INPLASY

PROTOCOL

To cite: Wei et al. Performance of resistive index and semiquantitative power doppler ultrasound score in predicting acute kidney injury: A metaanalysis of prospective studies. Inplasy protocol 202220101. doi: 10.37766/inplasy2022.2.0101

Received: 23 February 2022

Published: 23 February 2022

Corresponding author: Qiong Wei

1397726372@qq.com

Author Affiliation:

PLA 983rd Hospital

Support: The author(s) received no spec.

Review Stage at time of this submission: Completed but not published.

Conflicts of interest:

None declared.

\section{Performance of resistive index and semi-quantitative power doppler ultrasound score in predicting acute kidney injury: A meta-analysis of prospective studies}

Wei, Q1; Zhu, Y2; Zhen, W3; Zhang, X4; Shi, Z5; Zhang, L6; Zhou, J7.

Review question / Objective: This study aimed to assess the predictive value of the renal resistive index (RRI) and power Doppler ultrasound (PDU) on subsequent acute kidney injury (AKI) risk using a meta-analytic approach.

Condition being studied: No studies have compared the predictive performance of the RRI and PDU for AKI in patients with critical illnesses.

Eligibility criteria: Studies were included if the following inclusion criteria were met: (1) with critically ill or postoperative patients; (2) utilized the RRI or PDU; (3) incidence of $\mathrm{AKI}$, and the results of true positive, false positive, false negative, true negative, or data that could be used to calculate these values; and (4) had a prospective study design.

INPLASY registration number: This protocol was registered with the International Platform of Registered Systematic Review and Meta-Analysis Protocols (INPLASY) on 23 February 2022 and was last updated on 23 February 2022 (registration number INPLASY202220101).

\section{INTRODUCTION}

Review question / Objective: This study aimed to assess the predictive value of the renal resistive index (RRI) and power Doppler ultrasound (PDU) on subsequent acute kidney injury (AKI) risk using a metaanalytic approach.

Rationale: The parameters included the sensitivity, specificity, positive and negative likelihood ratios (PLR and NLR), diagnostic 
odds ratio (DOR), and area under the receiver operating characteristic curves (AUC).

Condition being studied: No studies have compared the predictive performance of the RRI and PDU for AKI in patients with critical illnesses

\section{METHODS}

Search strategy: "acute kidney injury" AND ("Doppler" OR "ultrasound").

Participant or population: Critically ill or post-operative patients.

Intervention: RRI or PDU.

Comparator: Gold standard.

Study designs to be included: Prospective study.

Eligibility criteria: Studies were included if the following inclusion criteria were met: (1) with critically ill or post-operative patients; (2) utilized the RRI or PDU; (3) incidence of $A K I$, and the results of true positive, false positive, false negative, true negative, or data that could be used to calculate these values; and (4) had a prospective study design.

Information sources: The databases of PubMed, EmBase, and the Cochrane library were systematically searched for studies from inception through August 2021. We also reviewed related review articles and original research articles to identify additional included studies.

Main outcome(s): incidence of AKI, and the results of true positive, false positive, false negative, true negative, or data that could be used to calculate these values.

Quality assessment / Risk of bias analysis: The Quality Assessment of Diagnostic Accuracy Studies 2 was applied to assess the quality of the selected studies using the following items: risk of bias (patient selection, index test, reference standard, flow and timing) and applicability concerns (patient selection, index test, reference standard).

Strategy of data synthesis: The number of true positives, false negatives, false positives, and true negatives was presented in each original study. The diagnostic odds ratio (DOR) and the area under the receiver operating characteristic curve (AUC) were then assessed to find the overall accuracy. The sensitivity, specificity, positive likelihood ratio (PLR), and negative likelihood ratio (NLR) were also assessed. The pooled parameters were calculated using the bivariate generalized linear mixed model and random-effects model. The heterogeneity across the included studies were assessed using the 12 and $Q$ statistic, and significant heterogeneity was considered as $12>50.0 \%$ or $P<0.10$. The indirect comparison of predictive performance between the RRI and PDU were illustrated, and the ratio with 95\% confidence interval $(\mathrm{Cl})$ between the RRI and PDU was calculated. If a $95 \% \mathrm{Cl}$ for the ratio included the value of 1 , there was insufficient evidence to conclude that the predictive performance of the RRI and PDU were significantly different.

Subgroup analysis: Subgroup analyses for the predictive parameters were performed based on the country, mean age of patients, proportion of males, and disease status, and the differences between the RRI and PDU or between subgroups were also determined.

Sensitivity analysis: Sensitivity analyses were performed by sequential removing individual study.

Language: No restriction were placed on published language.

Country(ies) involved: China.

Keywords: resistive index; Doppler Ultrasound; Kidney; Injury; Meta-Analysis.

Contributions of each author:

Author 1 - Qiong Wei.

Email: 1397726372@qq.com

Author 2 - Yu Zhu. 
Email: 308294233@qq.com

Author 3 - Weifeng Zhen.

Email: 90375339@qq.com

Author 4 - Xiaoning Zhang.

Email: xiaoningzh@sina.com

Author 5 - Zhenhua Shi.

Email: szhhaiwangxing@163.com

Author 6 - Ling Zhang.

Email: 37746187@qq.com

Author 7 - Jiuju Zhou.

Email: 316772269@qq.com 